\title{
Can the Utility of Anonymized Data be Used for Privacy Breaches?
}

\author{
RAYMOND CHI-WING WONG, The Hong Kong University of Science and Technology \\ ADA WAI-CHEE FU, The Chinese University of Hong Kong \\ KE WANG, Simon Fraser University \\ PHILIP S. YU, University of Illinois at Chicago \\ JIAN PEI, Simon Fraser University
}

\begin{abstract}
Group based anonymization is the most widely studied approach for privacy-preserving data publishing. Privacy models/definitions using group based anonymization includes $k$-anonymity, $l$-diversity, and $t$-closeness, to name a few. The goal of this article is to raise a fundamental issue regarding the privacy exposure of the approaches using group based anonymization. This has been overlooked in the past. The group based anonymization approach by bucketization basically hides each individual record behind a group to preserve data privacy. If not properly anonymized, patterns can actually be derived from the published data and be used by an adversary to breach individual privacy. For example, from the medical records released, if patterns such as that people from certain countries rarely suffer from some disease can be derived, then the information can be used to imply linkage of other people in an anonymized group with this disease with higher likelihood. We call the derived patterns from the published data the foreground knowledge. This is in contrast to the background knowledge that the adversary may obtain from other channels, as studied in some previous work. Finally, our experimental results show such an attack is realistic in the privacy benchmark dataset under the traditional group based anonymization approach.
\end{abstract}

Categories and Subject Descriptors: H.2.0 [Database Management]: General

General Terms: Algorithms, Experimentation, Security

Additional Key Words and Phrases: Privacy preservation, data publishing, $l$-diversity, $k$-anonymity

ACM Reference Format:

Wong, R. C.-W., Fu, A. W.-C., Wang, K., Yu, P. S., Pei, J. 2011. Can the utility of anonymized data be used for privacy breaches? ACM Trans. Knowl. Discov. Data 5, 3, Article 16 (August 2011), 24 pages.

DOI $=10.1145 / 1993077.1993080$ http://doi.acm.org/10.1145/1993077.1993080

\section{INTRODUCTION}

A major technique used in privacy-preserving data publishing is group based anonymization, whereby records in the given relation are partitioned into groups and each group must ensure some property such as diversity so as to satisfy the privacy requirement while maintaining sufficient data utility. There are many privacy models associated with group based anonymization, such as $k$-anonymity [Nergiz and Clifton 2007; Sweeney 2002], $l$-diversity [Machanavajjhala et al. 2006], $t$-closeness [Li and Li 2007], $(k, e)$-anonymity [Zhang et al. 2007], Injector [Li and Li 2008] and

The research of Raymond Chi-Wing Wong was supported by HKRGC GRF 621309. The research of Ke Wang was supported by a Discovery Grant from NSERC. The research of Philip S. Yu was supported by US NSF through grants IIS-0914934, OISE-0968341, and OIA-0963278.

Author's address: R. C.-W. Wong; email: raywong@cse.ust.hk.

Permission to make digital or hard copies of part or all of this work for personal or classroom use is granted without fee provided that copies are not made or distributed for profit or commercial advantage and that copies show this notice on the first page or initial screen of a display along with the full citation. Copyrights for components of this work owned by others than ACM must be honored. Abstracting with credit is permitted. To copy otherwise, to republish, to post on servers, to redistribute to lists, or to use any component of this work in other works requires prior specific permission and/or a fee. Permission may be requested from Publications Dept., ACM, Inc., 2 Penn Plaza, Suite 701, New York, NY 10121-0701, USA, fax +1 (212) 869-0481, or permissions@acm.org.

(c) 2011 ACM 1556-4681/2011/08-ART16 $\$ 10.00$

DOI 10.1145/1993077.1993080 http://doi.acm.org/10.1145/1993077.1993080 


\begin{tabular}{c|c|c|c|}
\multicolumn{4}{c}{ Table I. An Example } \\
\cline { 2 - 4 } Name & Nationality & Zipcode & Disease \\
\hline \multirow{3}{*}{$\begin{array}{c}\text { Alex } \\
\text { Bob }\end{array}$} & Malaysian & 45501 & Heart Disease \\
\cline { 2 - 4 } & Japanese & 45502 & Flu \\
\cline { 2 - 4 } & Japanese & 55503 & Flu \\
\cline { 2 - 4 } & Japanese & 55504 & Stomach Virus \\
\cline { 2 - 4 } & Chinese & 66601 & HIV \\
\cline { 2 - 4 } & Japanese & 66601 & Diabetes \\
\cline { 2 - 4 } & Indian & 77701 & Flu \\
\cline { 2 - 4 } & Singaporean & 77701 & Diabetes \\
\cline { 2 - 4 } & $\ldots$ & $\ldots$ & $\ldots$ \\
\cline { 2 - 4 }
\end{tabular}

$m$-confidentiality [Wong et al. 2007]. It seems that this technique is sound for privacypreserving data publishing. However, when examined more carefully, it suffers from one fundamental privacy violation problem, which has been overlooked in the past. The main cause of this problem is that the utility maintained in the anonymized table can help an adversary to breach individual privacy.

In the literature, background knowledge [Kifer and Gehrke 2006; Li and Li 2008; Machanavajjhala et al. 2006; Martin et al. 2007; Wong et al. 2007] such as the rarity of a disease among a certain ethnic group or the pattern of age or gender for a disease can often be used by an adversary. In this article, we show that such knowledge can be mined from the published data or the anonymized data. In fact, one of the purposes of data publishing is for data mining, which is mainly about the discovery of patterns from the published data.

Let us illustrate the problem with an example. Suppose a table $T$ is to be anonymized for publication. Table $T$ has two kinds of attributes, quasi-identifier (QI) attributes and a sensitive attribute.

The QI attributes can be used as an identifier in the table. Sweeney [2002] points out that in the United States, most individuals can be uniquely identified by QI attributes, namely birthdate, zipcode, and gender. Information about the QI attributes can often be obtained from some external tables such as a voter registration list. An example of a voter registration list is shown in Table II. In this table, there are three attributes, Name, Nationality, and Zipcode. Assume that the table contains information about a set of individuals, and the information about each such individual is contained in exactly one tuple. We also say that the individual owns the tuple. Assume attributes Nationality and Zipcode are the two QI attributes. Then the adversary can uniquely identify the tuple for Alex with the attributes Nationality and Zipcode.

The sensitive attribute contains some sensitive values. For example, if $T$ is Table I, the sensitive attribute is Disease, which contains sensitive values such as Heart Disease and HIV. Assume that each tuple in the table is owned by an individual and each individual owns at most one tuple.

After the anonymization, we publish the anonymized dataset $T^{*}$, which consists of a set of QI-groups, where each QI-group is a set of tuples linked with a multiset of sensitive values. Depending on the anonymization mechanism, each QI-group may correspond to either a set of quasi-identifer (QI) values or a single generalized QI value. An attribute GID is added for the ID of the QI-group. We shall refer to a QI-group by its GID. Such group-based anonymization is commonly adopted in the literature of data publishing [Aggarwal et al. 2005; LeFevre et al. 2005; Li and Li 2007, 2008; Wong et al. 2007; Xiao and Tao 2006] (including $k$-anonymity, $l$-diversity, $t$-closeness and a vast number of other privacy models). The linkage between individual records and the sensitive attribute in each QI-group must be broken. One way to break the linkage is 
Table II. Voter Registration List

\begin{tabular}{|c|c|c|}
\hline Name & Nationality & Zipcode \\
\hline Alex & Malaysian & 45501 \\
\hline Bob & Japanese & 45502 \\
\hline Chris & Japanese & 55503 \\
\hline David & Japanese & 55504 \\
\hline Emily & Chinese & 66601 \\
\hline Fred & Japanese & 66601 \\
\hline Gary & Indian & 77701 \\
\hline Henry & Singaporean & 77701 \\
\hline$\ldots$ & $\ldots$ & $\ldots$ \\
\hline
\end{tabular}

Table III. A 2-Diverse Dataset Anonymized from Table I by Bucketization

\begin{tabular}{|c|c|c|}
\hline Nationality & Zipcode & GID \\
\hline Malaysian & 45501 & $Q I_{1}$ \\
\hline Japanese & 45502 & $Q I_{1}$ \\
\hline Japanese & 55503 & $Q I_{2}$ \\
\hline Japanese & 55504 & $Q I_{2}$ \\
\hline Chinese & 66601 & $Q I_{3}$ \\
\hline Japanese & 66601 & $Q I_{3}$ \\
\hline Indian & 77701 & $Q I_{4}$ \\
\hline Singaporean & 77701 & $Q I_{4}$ \\
\hline$\ldots$ & $\ldots$ & $\ldots$ \\
\hline
\end{tabular}

(a) QI table.

\begin{tabular}{|c|c|}
\hline GID & Disease \\
\hline$Q I_{1}$ & Heart Disease \\
\hline$Q I_{1}$ & Flu \\
\hline$Q I_{2}$ & Flu \\
\hline$Q I_{2}$ & Stomach Virus \\
\hline$Q I_{3}$ & HIV \\
\hline$Q I_{3}$ & Diabetes \\
\hline$Q I_{4}$ & Flu \\
\hline$Q I_{4}$ & Diabetes \\
\hline$\ldots$ & $\ldots$ \\
\hline
\end{tabular}

(b) Sensitive table.

bucketization: forming two tables, called the QI table (Table III(a)) for the QI attributes and the sensitive table (Table III(b)) for the sensitive attribute. These two tables form the anonymized dataset $T^{*}$.

For illustration, a simplified setting of the $l$-diversity model [Machanavajjhala et al. 2006 ] is used as a privacy requirement for published data $T^{*}$. This simplified setting has been adopted many followup papers [Li et al. 2009; Xiao and Tao 2006, 2007; Wong et al. 2007] due to its lucid illustration of the concept behind $l$-diversity. A QI-group is said to be l-diverse or satisfy l-diversity if in the QI-group the number of occurrences of any sensitive value is at most $1 / l$ of the group size. A table satisfies $l$-diversity (or it is $l$-diverse) if all QI-groups in it are $l$-diverse. Table I is anonymized to Table III by bucketization. The first group containing the first two tuples is given a GID $Q I_{1}$. The GID for each group is shown in Table III. The intention is that each individual cannot be linked to a disease with a probability of more than 0.5 . However, does this table protect individual privacy sufficiently?

Let us examine the QI-group $Q I_{1}$ as shown in Table III. In $Q I_{1}$, Heart Disease and Flu are values of the sensitive attribute Disease. It seems that each of the two individuals, Alex and Bob, in this group has a 50\% chance of linking to Heart Disease (Flu). The reason why the chance is interpreted as $50 \%$ is that the analysis is based on this group locally without any additional information.

However, from the entire published table containing multiple groups, the adversary may discover some interesting patterns globally. For example, suppose the published table consists of many QI-groups like $Q I_{2}$ with all Japanese with no occurrence of Heart Disease. At the same time, there are many QI-groups like $Q I_{3}$ containing some Japanese without Heart Disease. The pattern that Japanese rarely suffer from Heart 
Disease can be uncovered. Note that it is very likely that such anonymized data is published by conventional anonymization methods, given the fact that Heart Disease occurs rarely among Japanese. With the pattern uncovered, the adversary can say that Bob, being Japanese, has less chance of having Heart Disease. S/he can deduce that Alex, being a Malaysian, has a higher chance of having Heart Disease. The intended $50 \%$ threshold is thus violated.

\subsection{Foreground Knowledge Attack}

The anonymized data can be seen as imprecise or uncertain data [Burdick et al. 2005, 2007], and an adversary can uncover interesting patterns since the published data must maintain high data utility [Wong et al. 2007; Xiao and Tao 2006; Zhang et al. 2007]. We call the uncovered patterns the foreground knowledge (which is implicitly inside the table) in contrast to the background knowledge, studied by existing works [Li and Li 2007; Machanavajjhala et al. 2006; Wong et al. 2007; Zhang et al. 2007], which requires much adversary effort to obtain from somewhere outside the table. Since it is easy to obtain the foreground knowledge from the anonymized dataset, most existing works suffer from privacy breaches.

In Table III, there are only two local possible worlds for assigning the disease values to the two individuals in $Q I_{1}$ : (1) $w_{1}$ : Alex is linked to Heart Disease and Bob is linked to Flu and (2) $w_{2}$ : Alex is linked to Flu and Bob is linked to Heart Disease. To construct a probability distribution over the domain of the real world, a simplest definition is based on the assumption that all the possible worlds are equally likely, or each world has the same probability.

If we publish a group $Q I_{1}$ alone, the random world assumption is a good principle in the absence of other information. However, when several groups are published together as is typically the case, the groups with Japanese contribute to a statement that their members are not likely linked to Heart Disease. This statement means that the probability (or weight) of the possible world $w_{1}$ is much greater than that of $w_{2}$.

Most previous privacy works such as $l$-diversity [Machanavajjhala et al. 2006], $t$ closeness [Li and Li 2007], $(k, e)$-anonymity [Zhang et al. 2007] and $m$-confidentiality [Wong et al. 2007] adopt the random world assumption locally. In this article, the source of attack of the adversary is to apply the more complete model of the weighted possible worlds. We call this kind of attack foreground knowledge attack.

\subsection{Generalization-Based Anonymization}

This example shows that the foreground knowledge attack appears in a table generated by bucketization. Although the example is based on bucketization anonymization, the same issue arises with a generalization based method [Machanavajjhala et al. 2006; Wong et al. 2006; $\mathrm{Li}$ and $\mathrm{Li}$ 2007]. The reason is that the adversary has at his/her disposal the external table with which he/she may be able to look up the details of individuals who are mapped to a QI-group. For example, if the QI values of $Q I_{4}$ in Table III are generalized to $\{$ Asian, $777 * *\}$, and Gary and Henry are the only Asians with a Zipcode of $777^{* *}$ in the external table, Table II, then an adversary can determine that they are the owners of the two tuples in $Q I_{4}$. Hence, the exact QI values of \{Indian, 77701\} and \{Singaporean, 77701\} will be disclosed for $Q I_{4}$, and the data for $Q I_{4}$ becomes as detailed as that from bucketization. Sweeney [2002] points out that in the United States, $87 \%$ of individuals can be uniquely identified by QI attributes, namely birthdate, zipcode, and gender, and therefore such information disclosure is not uncommon. Once such details are determined, the adversary can determine the revised probabilities. It is worth mentioning that it is more difficult for an adversary to perform the foreground knowledge attack on the table generated by generalization 
Table IV. A 2-Diverse Dataset Anonymized from Table I by Global Recoding

\begin{tabular}{|c|c|c|}
\hline Nationality & Zipcode & Disease \\
\hline Asian & $455^{* *}$ & Heart Disease \\
\hline Asian & $455^{* *}$ & Flu \\
\hline Asian & $555^{* *}$ & Flu \\
\hline Asian & $555^{* *}$ & Stomach Virus \\
\hline Asian & $666^{* *}$ & HIV \\
\hline Asian & $666^{* *}$ & Diabetes \\
\hline Asian & $777^{* *}$ & Flu \\
\hline Asian & $777^{* *}$ & Diabetes \\
\hline$\ldots$ & $\ldots$ & $\ldots$ \\
\hline
\end{tabular}

Table V. A 2-Diverse Dataset Anonymized from Table I by Local Recoding

\begin{tabular}{|c|c|c|}
\hline Nationality & Zipcode & Disease \\
\hline$\{$ Malaysian, Japanese $\}$ & $45501-45502$ & Heart Disease \\
\hline$\{$ Malaysian, Japanese $\}$ & $45501-45502$ & Flu \\
\hline Japanese & $55503-55504$ & Flu \\
\hline Japanese & $55503-55504$ & Stomach Virus \\
\hline$\{$ Chinese, Japanese $\}$ & 66601 & HIV \\
\hline$\{$ Chinese, Japanese $\}$ & 66601 & Diabetes \\
\hline$\{$ Indian, Singaporean $\}$ & 77701 & Flu \\
\hline$\{$ Indian, Singaporean $\}$ & 77701 & Diabetes \\
\hline$\ldots$ & $\ldots$ & $\ldots$ \\
\hline
\end{tabular}

compared with the table generated by bucketization. This is because in some cases, it is more likely that a given individual such as Alex can be mapped to multiple QIgroups in the table generated by generalization than to a single QI-group in the table generated by bucketization.

In the literature, there are two kinds of generalization techniques, namely global recoding and local recoding. We want to emphasize that the foreground knowledge attack occurs in the table generated by either global recoding or local recoding. Under global recoding, all occurrences of a single attribute value are recoded to the same value. Table IV shows a 2-diverse dataset anonymized from Table I by global recoding. With the external table (Table II), an adversary can figure out the original values of each tuple in each group in Table IV. For example, the QI values for the first two records must be $\{$ Malaysian, 45501\} and \{Japanese, 45502\}. Thus, foreground knowledge attack is valid. Under local recoding, occurrences of the same value of an attribute may be recoded to different values. Table $\mathrm{V}$ shows a 2-diverse dataset anonymized from Table I by local recoding. Note that notation \{Malaysian, Japanese\} means that this value is either Malaysian or Japanese. Similar to global recoding, once the adversary can figure out the original QI value of each tuple, the same principle for foreground knowledge attack can be applied. Note that since global recoding often incurs a higher information loss than local recoding, it is more resistent to foreground knowledge attack. The major focus of this article is to study a new form of attack. For ease of illustration, we will show how the attack can be successful for the case of bucketization. 


\subsection{Contributions}

Our contributions can be summarized as follows. First, we define and study data anonymization issues in data publication by considering a foreground knowledge attack. Our concept of the foreground knowledge attack was derived independently of Kifer [2009]; the paper by Kifer, and this article were written concurrently. Second, we show how an adversary can breach privacy by computing the probability that an individual is linked to a sensitive value by using foreground knowledge.

Finally, we have conducted experiments to show how the adversary can succeed in foreground knowledge in different anonymization schemes, including Anatomy [Xiao and Tao 2006], MASK [Wong et al. 2007], and Injector [Li and Li 2008], and also under different privacy requirements such as $t$-closeness [Li and $\mathrm{Li} 2007$ ].

We emphasize that, similar to $l$-diversity, most group-based anonymization algorithms [Li and Li 2007, 2008; Wong et al. 2007; Xiao and Tao 2006] by bucketization also suffer from possible privacy breaches due to the utility of the published table. We believe that this work is significant in pointing out this overlooked issue, and that follow-up work would need to deter foreground knowledge attack.

The rest of the article is organized as follows. Section 2 formulates the problem. Section 3 describes how an adversary can breach individual privacy with the foreground knowledge obtained from the anonymized data. Section 4 shows how an adversary can obtain the foreground knowledge from the anonymized data. An empirical study is reported in Section 5. Section 6 reviews the related work. The article is concluded in Section 7.

\section{PROBLEM DEFINITION}

Let $T$ be a table. We assume that one of the attributes is a sensitive attribute $X$, where some values of this attribute should not be linkable to any individual. The value of the sensitive attribute of a tuple $t$ is denoted by $t . X$. A quasi-identifier (QI) is a set of attributes of $T$, namely $A_{1}, A_{2}, \ldots, A_{q}$, that may serve as identifiers for some individuals. Each tuple in the table $T$ is related to one individual and no two tuples are related to the same individual.

Let $P$ be a partition of table $T$. We give a unique ID called GID to this partition $P$ and append an additional attribute called GID to this partition where each tuple in $P$ has the same GID value. Existing group-based anonymization defines a function $\beta$ on $P$ to form a QI-group such that the linkage between the QI attributes and the sensitive attribute in the QI-group is lost. There are two methods in the literature for this task. One is generalization by generalizing all QI values to the same value. The other is bucketization by forming two tables, called the QI table and the sensitive table, where $P$ is projected on all QI attributes and attribute GID to form the QI table, and on the sensitive attribute and attribute GID to form the sensitive table. A table $T$ is anonymized to a dataset $T^{*}$ if $T^{*}$ is formed by first partitioning $T$ into a number of partitions, then forming a QI-group from each partition by $\beta$ and finally inserting each QI-group into $T^{*}$. For example, Table I is anonymized to Table III by bucketization.

We assume that there is a mapping which maps each tuple in $T$ to a QI-group in $T^{*}$. For example, the first tuple $t_{1}$ in Table I is mapped to QI-group $Q I_{1}$.

In the following, we focus on discussing the anonymized table generated by bucketization.

In the literature [Li and $\mathrm{Li}$ 2007, 2008; Wong et al. 2007; Xiao and Tao 2006], it is assumed that the knowledge of the adversary includes, (1) the published dataset $T^{*}$, (2) the QI value of a target individual, and (3) an external table $T^{e}$ such as the voter registration list that helps to map QIs to individuals [Sweeney 2002; LeFevre et al. 2005]. We also follow these assumptions in our analysis. 
Table VI. A Global Distribution of Attribute "Nationality"

for Our Motivating Example

\begin{tabular}{|c|c|c|}
\hline$p()$ & Heart Disease & Not Heart Disease \\
\hline Malaysian & 0.1 & 0.9 \\
Japanese & 0.003 & 0.997 \\
Chinese & 0.05 & 0.95 \\
\hline
\end{tabular}

The aim of privacy-preserving data publishing is to deter any attack from an adversary on linking an individual to a certain sensitive value. The data publisher would try to limit the probability that such a linkage could be established. Let us consider an arbitrary sensitive value $x$ for the analysis. We denote any value in $X$ that is $\operatorname{not} x$, by $\bar{x}$.

In this article, we consider that an adversary can obtain additional information from the published dataset $T^{*}$ in the form of global distribution, which can lead to an individual privacy breach. In the example in Section 1, we can mine from the published table that the chance of Japanese suffering from Heart Disease is low compared with Malaysians. This pattern is from the global distribution for the attribute set \{ "Nationality" $\}$.

Each possible value in attribute "Nationality" is called a signature. There are three possible signatures in our example: "Japanese," "Malaysian," and "Chinese." In general, there are other attribute sets, such as \{ "Gender," "Nationality"\}, with their correspondence global distributions.

Definition 2.1 (Signature). Let $T^{*}$ be the published dataset. Given a QI attribute set $\mathcal{A}$ with $r$ attributes $A_{1}, \ldots, A_{r}$. A signature $s$ of $\mathcal{A}$ is a set of attribute-value pairs $\left(A_{1}, v_{1}\right), \ldots,\left(A_{r}, v_{r}\right)$ that appear in the published dataset $T^{*}$, where $A_{i}$ is a QI attribute and $v_{i}$ is a value. A tuple $t$ in $T^{*}$ is said to match $s$ if $t . A_{i}=v_{i}$ for all $i=1,2, \ldots, r$.

For example, a signature $s$ can be $\{$ ("Nationality," "Malaysian"), ("Gender," "Male") $\}$ if the attribute set $\mathcal{A}$ is $\{$ "Nationality," "Gender" $\}$. For convenience, we often drop the attribute names in a signature, and thus we refer to \{"Malaysian," "Male"\} instead of $\left\{\right.$ ("Nationality," "Malaysian"), ("Gender," "Male") . The first tuple $t_{1}$ in Table III(a) matches \{"Malaysian"\} but the second tuple does not.

Definition 2.2 (Sample Space $\Omega(s, x)$ ). The sample space $\Omega(s, x)$ for a signature $s$ and a sensitive value $x$ is a set of two elements: (1) $s$ is linked to $x$ and (2) $s$ is not linked to $x$ (or $s$ is linked to $\bar{x}$ ).

Consider an arbitrary sensitive value "Heart Disease." Assume that Table VI shows the global distribution of attribute set \{"Nationality"\}, which consists of the probabilities that a Japanese, a Malaysian, or a Chinese person is linked to Heart Disease. Each such probability in the table is called a global probability. The global probabilities are based on sample spaces for different signatures.

Definition 2.3 (Global Distribution). Given an attribute set $\mathcal{A}$, the global distribution $G$ of $\mathcal{A}$ contains a set of entries $(s: x, p)$ for each possible signature $s$ of $\mathcal{A}$, where $p$ is equal to $p(s: x)$, which denotes the probability that a tuple matching signature $s$ is linked to $x$, given the published dataset $T^{*}$.

For example, if $G$ contains ("Japanese":"Heart Disease," 0.003) and ("Malaysian": "Heart Disease," 0.1), then the probability that a Japanese patient is linked to Heart Disease is equal to 0.003 while that of a Malaysian patient is 0.1 . 
The global distribution $G$ derived from the published dataset $T^{*}$ is called the foreground knowledge. We will describe how the adversary derives $G$ from the published table.

Problem 1 (Foreground Knowledge). Given any arbitrary attribute set $\mathcal{A}$, we want to find the global distribution $G$ of $\mathcal{A}$ from the published dataset $T^{*}$.

From Section 1, we show that with the global distribution $G$ of attribute set \{"Nationality"\}, we can deduce that the chance of Alex, a Malaysian, suffering from Heart Disease is high. Let $t$ be Alex and $x$ be Heart Disease. The chance can be formulated by $p(t: x)$, the probability that $t$ is linked to $x$ given $G$.

Problem 2 (Privacy Breach). Given a published dataset $T^{*}$, for any individual $t$, and any sensitive value $x$, we want to determine whether the probability that $t$ is linked to $x$, denoted by $p(t: x)$, is greater than $1 / r$. Individual $t$ is said to suffer from privacy breaches if the probability is greater than $1 / r$.

We should point out here that knowing that a Japanese person has a 0.003 probability of Heart Disease does not lead to a conclusion that a Japanese person $t$ in a QI-group $Q I$ in $T^{*}$ also has a 0.003 chance of Heart Disease. A very simple counter example is when the QI-group does not contain a record with Heart Disease. In this example, the probability is obviously 0 . However, how to derive such a probability in general is nontrivial and will be the main focus in the next two sections. Another point to clarify is that Problems (1) and (2) need to be solved as one problem. The global distribution $G$ is dependent on the probabilities $p(t: x)$ and vice versa. This is because $T^{*}$ consists of QI-groups in which the probabilities $p(t: x)$ from each group contribute to $G$, and conversely, $G$ in turn affects $p(t: x)$.

In this article, we study Problems (1) and (2). In Section 3, we will first describe how we solve Problem (2), assuming that we are given the foreground knowledge. Then, in Section 4, we will describe how we can mine the foreground knowledge from the published dataset $T^{*}$ for Problem (1). We shall show that the two problems are intertwined and they are solved as one problem.

\section{FINDING PRIVACY BREACHES}

In this section, we derive a formula to calculate the probability that an individual $t$ is linked to a sensitive value $x$. The major idea is similar to what we discussed in Section 1. Consider an individual $t$ (e.g., Alex) in a QI-group $Q I_{k}$ of the published table $T^{*}$. Suppose $Q I_{k}$ contains $N$ individuals and $N$ values in the sensitive attribute. We can enumerate all possible assignments (or possible worlds) between a set of $N$ individuals and a set of $N$ values for $Q I_{k}$. In Section 1 , since $N$ is equal to 2, there are two possible worlds for $Q I_{k}$. Assume that $G$ is the global distribution of a certain attribute set $\mathcal{A}$ (e.g., $\{$ Nationality\}). According to $G$, different possible worlds have different probabilities. Finally, according to the probabilities of these possible worlds, we calculate the probability that $t$ is linked to a sensitive value $x$.

Formally, we derive the formula as follows. We assume that the attack is based on the linkage of an attribute set $\mathcal{A}$ to a sensitive value $x$. We denote by $\bar{x}$ any value not equal to $x$. In this section, we assume that the global distributions $G$ for $\mathcal{A}$ and $x$ have been determined and we show how an adversary can use $G$ to find privacy breaches. How the global distributions can be derived is explained in Section 4 .

Consider the motivating example in Section 1. In Table I, attribute "Nationality" contains "Malaysian", "Japanese," and "Chinese". Each value in "Nationality" is called a signature of attribute "Nationality". Thus, there are three possible signatures of a single attribute, namely "Nationality". In general, there are signatures of an attribute 
Table VII. Global Distribution

\begin{tabular}{|c|c|c|}
\hline$p()$ & $x$ & $\bar{x}$ \\
\hline$s_{1}$ & $f_{1}$ & $\overline{f_{1}}$ \\
$s_{2}$ & $f_{2}$ & $\overline{f_{2}}$ \\
$:$ & $:$ & $:$ \\
\hline
\end{tabular}

Table VIII. Notations

\begin{tabular}{|c|c|}
\hline$Q I_{k}$ & $\begin{array}{l}\text { a QI-group in the } \\
\text { anonymized dataset }\end{array}$ \\
\hline $\mathcal{A}$ & set of attributes e.g. $\{$ "Nationality", "Gender"\} \\
\hline$t_{1}, \ldots, t_{N}$ & tuples in an $A$-group \\
\hline$s_{1}, \ldots, s_{m}$ & $\begin{array}{l}\text { signatures for } \mathcal{A} \text {, e.g. }\{\text { "Malaysian", "Male" }\} \\
\text { multiple tuples } t_{j} \text { 's can map to the same } s_{i}\end{array}$ \\
\hline$x$ & a sensitive value \\
\hline $\bar{x}$ & any value not equal to $x$ \\
\hline$p\left(t_{j}: x\right)$ & probability that tuple $t_{j}$ is linked to value $x$ \\
\hline$p\left(s_{i}: x\right)$ & probability that signature $s_{i}$ is linked to $x$ \\
\hline$f_{i}$ & a simplified notation for $p\left(s_{i}: x\right)$ \\
\hline $\bar{f}_{i}$ & $1-f_{i}$ \\
\hline$w$ & $\begin{array}{l}\text { a possible world: an assignment of the tuples } \\
\text { in QI-group } Q I_{k} \text { to the sensitive values } x \text { and } \bar{x}\end{array}$ \\
\hline $\mathcal{W}_{k}$ & set of all possible worlds $w$ for $Q I_{k}$ \\
\hline $\mathcal{W}_{k}^{\left(t_{j}: x\right)}$ & $\begin{array}{l}\text { set of all possible worlds } w \text { in } \mathcal{W}_{k} \\
\text { in which } t_{j} \text { is assigned value } x \text {. }\end{array}$ \\
\hline$p(w)$ & $\begin{array}{l}\text { probability that } w \text { occurs given the anonymized } \\
\text { dataset and based on } \mathcal{A}\end{array}$ \\
\hline$p\left(w \mid Q I_{k}\right)$ & $\begin{array}{l}\text { conditional probability that } w \text { occurs given } \\
\text { QI-group } Q I_{k}\end{array}$ \\
\hline$\overline{p_{j, w}}$ & $\begin{array}{l}\text { the probability that } t_{j} \text { is linked to a value in the } \\
\text { sensitive attribute as specified in } w\end{array}$ \\
\hline $\mathcal{Q \mathcal { I }}_{s_{i}}$ & set of QI-groups containing tuples matching $s_{i}$ \\
\hline$Q I_{k}\left(s_{i}\right)$ & the set of tuples in $Q I_{k}$ matching $s_{i}$. \\
\hline$c_{k}\left(s_{i}: x\right)$ & $\begin{array}{l}\text { the expected number of tuples that match } s_{i} \\
\text { and are linked to } x \text { in the QI-group } Q I_{k}\end{array}$ \\
\hline
\end{tabular}

set containing multiple attributes. An example of an attribute set can be $\{$ Nationality, Zipcode\}. ("Malaysian", 5501) is a signature of \{Nationality, Zipcode\}.

Formally, suppose there are $m$ possible signatures for attribute set $\mathcal{A}$, namely $s_{1}, s_{2}, \ldots, s_{m}$. The global distribution $G$ of $\mathcal{A}$ is shown in Table VII. To simplify our presentation, the probability that $s_{i}$ is linked to $x(\bar{x}), p\left(s_{i}: x\right)\left(p\left(s_{i}: \bar{x}\right)\right)$, is denoted by $f_{i}\left(\bar{f}_{i}\right)$.

Given $G$, the formula for $p(t: x)$, the probability that a tuple $t$ is linked to sensitive value $x$, is derived here. Suppose $t$ belongs to QI-group $Q I_{k}$. For ease of reference, let us summarize the notations that we use in Table VIII.

Definition 3.1 (Sample Space $\Omega\left(Q I_{k}\right)$ ). Given a QI-group $Q I_{k}$ in the form of a set of tuples and a multiset of sensitive values $V$, we define a sample space $\Omega\left(Q I_{k}\right)$ for $Q I_{k}$ to 
be the set of all possible one-to-one assignments of the sensitive values $V$ to the tuples in $Q I_{k}$.

Definition 3.2 (Possible World). Consider a QI-group $Q I_{k}$ with $N$ tuples, namely $t_{1}, t_{2}, \ldots, t_{N}$, with sensitive values $\gamma_{1}, \gamma_{2}, \ldots \gamma_{N}$, where $\gamma_{i}$ is either $x$ or $\bar{x}$ for $i=1,2, \ldots, N$. A possible world $w$ for $Q I_{k}$ is a possible assignment mapping the tuples in set $\left\{t_{1}, t_{2}, \ldots, t_{N}\right\}$ to values in multiset $\left\{\gamma_{1}, \gamma_{2}, \ldots \gamma_{N}\right\}$ in $Q I_{k}$.

Thus, each element in $\Omega\left(Q I_{k}\right)$ is a possible world.

Definition 3.3 (Primitive Events, Projected Events). A mapping $t: \gamma$ from an individual or tuple $t$ to a sensitive value $\gamma(x$ or $\bar{x})$ is called a primitive event. Suppose $t$ matches signature $s$. Let us call an event for the corresponding signature, "s $s \gamma$," a projected event for $t$.

Hence, a primitive event $(t: x)$ is an event defined by a subset of $\Omega(Q)$ consisting of the possible worlds where $t$ is assigned to $x$. The probability of this event, $p(t: x)$, is a probability of interest for the adversary. A projected event is a corresponding event $(s: x)$, where $p(s: x)$ appears in the global distribution $G$.

Suppose that we are given a QI-group $Q I_{k}$ with a set of tuples and a multiset of sensitive values. For each possible world $w$ for $Q I_{k}$, according to the global distribution $G$ based on attribute set $\mathcal{A}$, we compute the probability $p(w)$ that $w$ occurs. The sample space for $p(w)$ consists of all the possible assignments of $x$ or $\bar{x}$ to a set of $N$ tuples with the same signatures as those in $Q I_{k}$.

Example 3.4. In our motivating example, consider the first QI-group $Q I_{1}$ and $x$ is "Heart Disease." There are two possible world for $Q I_{1}$ : (1) $w_{1}$ : Alex is linked to $x$ and Bob is linked to $\bar{x}$ and (2) $w_{2}$ : Alex is linked to $\bar{x}$ and Bob is linked to $x$. According to the global distribution of attribute "Nationality" as shown in Table VI, we would like to compute the probability that $w_{1}$ occurs, denoted by $p\left(w_{1}\right)$, and the probability that $w_{2}$ occurs. Consider $w_{1}$. From Table VI, we know that the probability that a Malaysian person is linked to $x$ is 0.1 and the probability that a Japanese person is linked to $\bar{x}$ is 0.997 . Since Alex is Malaysian, the probability that he is linked to $x$ is 0.1 . Similarly, since Bob is Japanese, the probability that he is linked to $\bar{x}$ is 0.997 . Similar to Machanavajjhala et al. [2006], Xiao and Tao [2006], and Wong et al. [2007], we assume that the linkage of a sensitive value to an individual is independent of the linkage of a sensitive value to another individual. For example, whether a Malaysian person suffers from Heart Disease is independent of whether a Japanese person suffers from Heart Disease. We conclude that $p\left(w_{1}\right)$ is equal to $0.1 \times 0.997=0.0997$. Similarly, $p\left(w_{2}\right)$ is equal to $0.003 \times 0.9=0.0027$.

We have just illustrated the major idea of computing $p(w)$ for a possible world $w$. In the following, we will give a formal derivation for computing $p(w)$.

Formally, suppose that in a possible world $w$ for $Q I_{k}$, tuple $t_{j}$ is linked to $\gamma$, where $\gamma$ is either $x$ or $\bar{x}$. Let $p_{j, w}$ be the probability that $t_{j}$ is linked to $\gamma$.

Note that we assume that the linkage of a sensitive value to an individual is independent of the linkage of a sensitive value to another individual. For a possible world $w$ for $Q I_{k}$, the probability that $w$ occurs is the product of the probabilities of the corresponding projected events for the tuples $t_{1}, \ldots t_{N}$ in $Q I_{k}$.

$$
p(w)=p_{1, w} \times p_{2, w} \times \ldots \times p_{N, w} .
$$

Suppose $t_{j}$ matches signature $s_{i}$. If $t_{j}$ is linked to $x$ in $w$, then $p_{j, w}=f_{i}$. Otherwise, $p_{j, w}=\bar{f}_{i}$.

$p(w)$ corresponds to the weight of $w$, which we mentioned in the introduction. 
We have just given a formal derivation of computing $p(w)$. Note that $p(w)$ considers the likelihood that $w$ occurs in the entire anonymized table but it does not consider any particular QI-group. In order to consider a particular QI-group $Q I_{k}$, in the following, we first illustrate how we derive a formula for computing the probability that $w$ occurs when we consider $Q I_{k}$ only, denoted by $p\left(w \mid Q I_{k}\right)$. After that, we give a formal derivation for this formula.

Example 3.5. From Example 3.4, we know that $p\left(w_{1}\right)=0.0997$ and $p\left(w_{2}\right)=0.0027$. Consider $Q I_{1}$. We know that there are only two possible worlds, namely $w_{1}$ and $w_{2}$, for $Q I_{1}$. The probability that $Q I_{1}$ occurs, given the anonymized table $T^{*}$, is equal to $0.0997+0.0027=0.1024$. Thus, the probability that $w_{1}$ occurs, given $Q I_{1}$, denoted by $p\left(w_{1} \mid Q I_{1}\right)$, is

$$
\frac{0.0997}{0.1024}=0.9736
$$

Similarly, $p\left(w_{2} \mid Q I_{1}\right)$ is equal to

$$
\frac{0.0027}{0.1024}=0.0264 \text {. }
$$

It is easy to verify that $p\left(w_{1} \mid Q I_{1}\right)+p\left(w_{2} \mid Q I_{1}\right)=1$.

Formally, the probability of $Q I_{k}$, given $T^{*}$, is the sum of the probabilities of all the possible worlds consistent with $T^{*}$ for $Q I_{k}$. Let the set of these worlds be $\mathcal{W}_{k}$. For $w \in \mathcal{W}_{k}$, we have

$$
p\left(w \mid Q I_{k}\right)=\frac{p(w)}{\sum_{w^{\prime} \in \mathcal{W}_{k}} p\left(w^{\prime}\right)} .
$$

It is easy to verify that $\sum_{w \in \mathcal{W}_{k}} p\left(w \mid Q I_{k}\right)=1$.

Our objective is to find the probability that an individual $t_{j}$ in $Q I_{k}$ is linked to a sensitive value $x$, denoted by $p\left(t_{j}: x\right)$.

Example 3.6. Consider that we are interested in knowing the probability that Alex in $Q I_{1}$ is linked to Heart Disease (i.e., $x$ ). There are two possible worlds for $Q I_{1}$ and there is only one possible world in which Alex is linked to $x\left(w_{1}\right)$. Let $t_{j}$ be Alex. The probability that Alex is linked to $x$, denoted by $p\left(t_{j}: x\right)$, is equal to $p\left(w_{1} \mid Q I_{1}\right)=0.9736$.

Formally, $p\left(t_{j}: x\right)$ is given by the sum of the conditional probabilities $p\left(w \mid Q I_{k}\right)$ of all the possible worlds $w$ where $t_{j}$ is linked to $x$.

$$
p\left(t_{j}: x\right)=\sum_{w \in \mathcal{W}_{k}^{\left(t_{j} ; x\right)}} p\left(w \mid Q I_{k}\right),
$$

where $\mathcal{W}_{k}^{\left(t_{j}: x\right)}$ is a set of all possible worlds $w$ in $\mathcal{W}_{k}$ in which $t_{j}$ is assigned value $x$.

One can verify that $p\left(t_{j}: x\right)+p\left(t_{j}: \bar{x}\right)=1$.

Example 3.7. Consider a QI-group $Q I_{k}$ in a published table $T^{*}$. Suppose there are four tuples, $t_{1}, t_{2}, t_{3}$, and $t_{4}$, and four sensitive values, $x, x, \bar{x}$, and $\bar{x}$ in $Q I_{k}$. Suppose the published table $T^{*}$ satisfies 2 -diversity.

Consider the global distribution $G$ based on a certain QI attribute set $\mathcal{A}$ that contains two possible signatures $s_{1}$ and $s_{2}$ as shown in Table IX(a).

Suppose $t_{1}, t_{2}, t_{3}$, and $t_{4}$ match signatures $s_{1}, s_{1}, s_{2}$, and $s_{2}$, respectively. There are six possible worlds $w$ as shown in Table IX(b). For example, the first row is the possible world $w_{1}$ with mapping $\left\{t_{1}: x, t_{2}: x, t_{3}: \bar{x}, t_{4}: \bar{x}\right\}$. The table also shows the probability $p(w)$ of the possible worlds. Take the first possible world $w_{1}$ for illustration. From 
Table IX. An Example Illustrating the Computation of $p\left(t_{j}: x\right)$

\begin{tabular}{|c|c|c|}
\hline$p()$ & $x$ & $\bar{x}$ \\
\hline$s_{1}$ & 0.5 & 0.5 \\
$s_{2}$ & 0.2 & 0.8 \\
\hline
\end{tabular}

(a) Global distribution

\begin{tabular}{|c|c|c|c|c|c|c|}
\hline$w$ & $\begin{array}{c}t_{1} \\
\left(s_{1}\right)\end{array}$ & $\begin{array}{c}t_{2} \\
\left(s_{1}\right)\end{array}$ & $\begin{array}{c}t_{3} \\
\left(s_{2}\right)\end{array}$ & $\begin{array}{c}t_{4} \\
\left(s_{2}\right)\end{array}$ & $p(w)$ & $p\left(w \mid Q I_{k}\right)$ \\
\hline$w_{1}$ & $x$ & $x$ & $\bar{x}$ & $\bar{x}$ & $0.5 \times 0.5 \times 0.8 \times 0.8=0.16$ & $0.16 / 0.33=0.48$ \\
\hline$w_{2}$ & $x$ & $\bar{x}$ & $x$ & $\bar{x}$ & $0.5 \times 0.5 \times 0.2 \times 0.8=0.04$ & $0.04 / 0.33=0.12$ \\
\hline$w_{3}$ & $x$ & $\bar{x}$ & $\bar{x}$ & $x$ & $0.5 \times 0.5 \times 0.8 \times 0.2=0.04$ & $0.04 / 0.33=0.12$ \\
\hline$w_{4}$ & $\bar{x}$ & $x$ & $x$ & $\bar{x}$ & $0.5 \times 0.5 \times 0.2 \times 0.8=0.04$ & $0.04 / 0.33=0.12$ \\
\hline$w_{5}$ & $\bar{x}$ & $x$ & $\bar{x}$ & $x$ & $0.5 \times 0.5 \times 0.8 \times 0.2=0.04$ & $0.04 / 0.33=0.12$ \\
\hline$w_{6}$ & $\bar{x}$ & $\bar{x}$ & $x$ & $x$ & $0.5 \times 0.5 \times 0.2 \times 0.2=0.01$ & $0.01 / 0.33=0.03$ \\
\hline
\end{tabular}

(b) $p(w)$ and $p\left(w \mid Q I_{k}\right)$

the global distribution in Table IX(a), $p\left(s_{1}: x\right)=0.5$ and $p\left(s_{2}: \bar{x}\right)=0.8$. Hence, $p\left(w_{1}\right)=0.5 \times 0.5 \times 0.8 \times 0.8=0.16$. The sum of probabilities $p(w)$ of all possible worlds from Table IX $(\mathrm{b})$ is equal to $0.16+0.04+0.04+0.04+0.04+0.01=0.33$. Consider $w_{1}$ again. Since $p\left(w_{1}\right)=0.16, p\left(w_{1} \mid Q I_{k}\right)=0.16 / 0.33=0.48$.

Suppose an adversary is interested in the probability that $t_{1}$ is linked to $x$. We obtain $p\left(t_{1}: x\right)$ as follows. $w_{1}, w_{2}$, and $w_{3}$, as shown in Table IX(b), contain " $t_{1}: x$ ". Thus, $p\left(t_{1}: x\right)$ is equal to the sum of the probabilities $p\left(w_{1} \mid Q I_{k}\right), p\left(w_{2} \mid Q I_{k}\right)$, and $p\left(w_{3} \mid Q I_{k}\right)$. $p\left(t_{1}: x\right)=0.48+0.12+0.12=0.72$, which is greater than 0.5 , the intended upper bound for 2-diversity that an individual is linked to a sensitive value.

Let $\left|Q I_{k}\right|$ be the size of the QI-group containing $t_{j}$ and $\left|\mathcal{W}_{k}\right|$ be the number of possible worlds in a QI-group $Q I_{k}$. We will generate $\left|\mathcal{W}_{k}\right|$ possible worlds. For each possible world, we calculate $p(w)$ and $p\left(w \mid Q I_{k}\right)$ in $O\left(\left|Q I_{k}\right|\right)$ time. Thus, the time complexity is $O\left(\left|Q I_{k}\right| \cdot\left|\mathcal{W}_{k}\right|\right)$.

The time complexity depends on two factors. One is $\left|Q I_{k}\right|$ and another is $\left|\mathcal{W}_{k}\right|$. (1) $\left|Q I_{k}\right|$ is bounded by the greatest size of the QI-group, which depends on the anonymization techniques. For example, $\left|Q I_{k}\right|$ is equal to $l$ or $l+1$ for algorithm Anatomy [Xiao and Tao 2006], which requires that each QI-group contains either $l$ or $l+1$ tuples. In our experiment, $\left|Q I_{k}\right|$ is at most 23 for algorithm MASK [Wong et al. 2007], where $l=2$. (2) $\left|\mathcal{W}_{k}\right|$ is equal to $C_{n}^{N}$, where $n$ is the number of tuples with $x$ in this QI-group of size $N$ and $C_{n}^{N}$ denotes the total number of possible ways of choosing $n$ objects from $N$ objects. Note that $\left|\mathcal{W}_{k}\right|$ is typically small because $n$ is usually equal to a small number. For algorithm Anatomy [Xiao and Tao 2006], as we discussed, $N$ (which corresponds to the size of the QI-group) is either $l$ or $l+1$. In this algorithm, since $x$ appears in the QI-group at most once, $n$ (which corresponds to the number of tuples with $x$ in this QI-group) is at most 1 . Thus, for each possible $x,\left|\mathcal{W}_{k}\right|$ is at most $l+1$. For Algorithm MASK [Wong et al. 2007], in our experiment, with $l=2$, the greatest frequency of $x$ in a QI-group is 8 . The size of this QI-group is $23 .\left|\mathcal{W}_{k}\right|$ is equal to $C_{8}^{23}=490,314$. When $l=10$, the greatest possible value of $\left|\mathcal{W}_{k}\right|$ is $140,364,532$. These values are small compared with the excessive number of possible worlds studied in uncertain data [Antova et al. 2007; Burdick et al. 2005, 2007; Cheng et al. 2008; Imielinski and Lipski 1984] (e.g., $10^{10^{6}}$ in Antova et al. [2007]). In the experimental setups in existing works [ $\mathrm{Li}$ and $\mathrm{Li}$ 2007, 2008; Machanavajjhala et al. 2006; Wong et al. 2007; Xiao and Tao 2006], $l \leq 10$. In other words, $\mathcal{W}_{k}$ can be generated within a reasonable time. 


\section{MINING FOREGROUND KNOWLEDGE}

We first describe how we find the global distribution $G$ of a certain attribute set $\mathcal{A}$ from the anonymized data in Section 4.1. Next, we introduce a pruning strategy to prune our search space of attribute sets in Section 4.2. Finally, we describe the algorithm for finding the global distribution of multiple attribute sets and discuss its complexity in Section 4.3.

\subsection{Foreground Knowledge}

In the previous section, we assumed that the values of $f_{i}$ are given. Here we consider how to derive $f_{i}$ from the published table $T^{*}$. We will develop $m$ equations involving the $m$ variables $f_{i}, 1 \leq i \leq m$.

Let the set of QI-groups in $T^{*}$ be $Q I_{1}, \ldots, Q I_{u}$. Let $Q I_{k}\left(s_{i}\right)$ be the set of tuples in $Q I_{k}$ matching signature $s_{i}$. For example, in Table III, let $s_{i}=\{$ "Malaysian" $\}$. Then, $Q I_{1}\left(s_{i}\right)$ contains only the first tuple.

Let $\mathcal{Q} \mathcal{I}_{s_{i}}$ be a set of QI-groups containing tuples that match $s_{i}$. That is, $\mathcal{Q} \mathcal{I}_{s_{i}}=$ $\left\{Q I_{k} \mid Q I_{k}\left(s_{i}\right) \neq \emptyset\right\}$.

$f_{i}$ is equal to the expected number of tuples that match $s_{i}$ and are linked to $x$ in $T^{*}$ divided by the number of tuples that match $s_{i}$ in $T^{*}$. Let $c_{k}\left(s_{i}: x\right)$ be the expected number of tuples that match $s_{i}$ and are linked to $x$ in the QI-group $Q I_{k}$. Then we can express $f_{i}$ as follows.

$$
f_{i}=\frac{\sum_{Q I_{k} \in \mathcal{Q} \mathcal{I}_{s_{i}}} c_{k}\left(s_{i}: x\right)}{\sum_{Q I_{k} \in \mathcal{Q} \mathcal{I}_{s_{i}}}\left|Q I_{k}\left(s_{i}\right)\right|} .
$$

The denominator is simply equal to the number of occurrences of $s_{i}$ in $T^{*}$, which can be easily found from the dataset. Let us consider the term $c_{k}\left(s_{i}: x\right)$ in the numerator.

Without additional knowledge to govern otherwise, we assume that the event that a tuple matching $s_{i}$ in $Q I_{k}$ is linked to $x$ is independent of the event that another tuple also matching $s_{i}$ in $Q I_{k}$ is linked to $x$. Then we have the following.

$$
c_{k}\left(s_{i}: x\right)=\left|Q I_{k}\left(s_{i}\right)\right| \times p\left(t_{j}: x\right),
$$

where $t_{j}$ is any tuple in $Q I_{k}$ matching $s_{i}$. Note that any $t_{j}$ in $Q I_{k}$ matching $s_{i}$ can be used here since all such $p\left(t_{j}: x\right)$ values are equal. Substitute Equations (3) and (2) into Equation (5) and we get

$$
c_{k}\left(s_{i}: x\right)=\left|Q I_{k}\left(s_{i}\right)\right| \times \sum_{w \in \mathcal{W}_{k}^{\left(t_{j} ; x\right)}} \frac{p(w)}{\sum_{w^{\prime} \in \mathcal{W}_{k}} p\left(w^{\prime}\right)} .
$$

Hence, $c_{k}\left(s_{i}: x\right)$ is expressed in terms of probabilities $p(w)$, which in turn are expressed in the $m$ variables $f_{i}$ (see Equation (1) where $p_{j, w}$ is equal to $f_{i}$ or $\bar{f}_{i}$ ). Here note that $\overline{f_{i}}=1-f_{i}$.

There are $m$ equations of the form of Equation (4) for the expression of $f_{i}, 1 \leq i \leq$ $m$. These equations involve $m$ variables, $f_{i}$. This is a classical problem of a system of simultaneous nonlinear equations, which occurs in many applications. It can be solved by conventional methods such as Newton's method and Bairstow's iteration. Since Newton's method [Chapra and Canale 2002] has been known to be effective and feasible, we choose this method for our study in this article.

Example 4.1. Given a table $T$ containing six tuples, $t_{1}, t_{2}, \ldots, t_{6}$, as shown in Table X. If the objective of the privacy requirement is 2-diversity, $T$ does not satisfy 2-diversity. Thus, an anonymized dataset $T^{*}$ in Table XI with three QI-groups, $Q I_{1}, Q I_{2}$, and $Q I_{3}$, is published. (For each sensitive value $x$ and each QI-group, the fraction of tuples with 


\begin{tabular}{|c|c|c|}
\hline $\mathcal{A}$ & $\ldots$ & $X$ \\
\hline$s_{1}$ & $\ldots$ & $x$ \\
\hline$s_{1}$ & $\ldots$ & $x$ \\
\hline$s_{1}$ & $\ldots$ & $\bar{x}$ \\
\hline$s_{2}$ & $\ldots$ & $\bar{x}$ \\
\hline$s_{2}$ & $\ldots$ & $\bar{x}$ \\
\hline$s_{2}$ & $\ldots$ & $\bar{x}$ \\
\hline
\end{tabular}

Table XI. An Example Illustrating the Computation of the Global Distribution

\begin{tabular}{c|c|c|c|}
\cline { 2 - 4 }$t$ & $\mathcal{A}$ & $\ldots$ & GID \\
\hline$t_{1}$ & $s_{1}$ & $\ldots$ & $Q I_{1}$ \\
\cline { 2 - 4 }$t_{2}$ & $s_{2}$ & $\ldots$ & $Q I_{1}$ \\
\cline { 2 - 4 }$t_{3}$ & $s_{1}$ & $\ldots$ & $Q I_{2}$ \\
\cline { 2 - 4 }$t_{4}$ & $s_{1}$ & $\ldots$ & $Q I_{2}$ \\
\cline { 2 - 4 }$t_{5}$ & $s_{2}$ & $\ldots$ & $Q I_{3}$ \\
\cline { 2 - 4 }$t_{6}$ & $s_{2}$ & $\ldots$ & $Q I_{3}$ \\
\hline
\end{tabular}

(a) QI Table

\begin{tabular}{|c|c|}
\hline GID & $X$ \\
\hline$Q I_{1}$ & $x$ \\
\hline$Q I_{1}$ & $\bar{x}$ \\
\hline$Q I_{2}$ & $x$ \\
\hline$Q I_{2}$ & $\bar{x}$ \\
\hline$Q I_{3}$ & $\bar{x}$ \\
\hline$Q I_{3}$ & $\bar{x}$ \\
\hline
\end{tabular}

(b) Sensitive Table

$x$ is at most 0.5 .) Note that $Q I_{3}$ satisfies 2-diversity. This is because since $\bar{x}$ corresponds to a value not equal to $x$, in $Q I_{3}$, the first $\bar{x}$ corresponds to a value $y$ and the second $\bar{x}$ corresponds to another value $z$.

Consider the global distribution of attribute set $\mathcal{A}$. There are two possible signatures based on $\mathcal{A}$, namely $s_{1}$ and $s_{2}$. Thus, we have two equations with two variables, namely $f_{1}$ and $f_{2}$, the probabilities in the global distribution $G$ of $\mathcal{A}$, as shown in Table VII.

Consider $f_{1}$. Since only QI-groups $Q I_{1}$ and $Q I_{2}$ contain the tuples matching $s_{1}$, $\mathcal{Q I _ { s _ { 1 } }}=\left\{Q I_{1}, Q I_{2}\right\}$.

$$
f_{1}=\left[\sum_{Q I_{k} \in \mathcal{Q} \mathcal{I}_{s_{1}}} c_{k}\left(s_{1}: x\right)\right] /\left[\sum_{Q I_{k} \in \mathcal{Q I}_{s_{1}}}\left|Q I_{k}\left(s_{1}\right)\right|\right] .
$$

$Q I_{1}$ contains one tuple $t_{1}$ matching $s_{1}$, and $Q I_{2}$ contains two tuples $t_{3}, t_{4}$, matching $s_{1}$, $\left|Q I_{1}\left(s_{1}\right)\right|=1$ and $\left|Q I_{2}\left(s_{1}\right)\right|=2$. Thus,

$$
f_{1}=\left[1 \times p\left(t_{1}: x\right)+2 \times p\left(t_{3}: x\right)\right] /(1+2) .
$$

Consider $Q I_{1}$. There are only two possible worlds, $w_{1}=\left\{t_{1}: x, t_{2}: \bar{x}\right\}$ and $w_{2}=\left\{t_{1}:\right.$ $\left.\bar{x}, t_{2}: x\right\}$. Note that $t_{1}$ and $t_{2}$ match signatures $s_{1}$ and $s_{2}$, respectively. $p_{1, w_{1}}=f_{1}, p_{2, w_{1}}=$ $\bar{f}_{2}, p_{1, w_{2}}=\bar{f}_{1}$ and $p_{2, w_{2}}=f_{2}$. Thus, $p\left(w_{1}\right)=p_{1, w_{1}} \times p_{2, w_{1}}=f_{1} \times \bar{f}_{2}$ and $p\left(w_{2}\right)=$ $p_{1, w_{2}} \times p_{2, w_{2}}=\overline{f_{1}} \times f_{2}$. We derive that

$$
p\left(t_{1}: x\right)=p\left(w_{1} \mid Q I_{1}\right)=f_{1} \overline{f_{2}} /\left(f_{1} \overline{f_{2}}+\overline{f_{1}} f_{2}\right) .
$$

Similarly, consider $Q I_{2}$. There are two possible worlds, $w_{3}=\left\{t_{3}: x, t_{4}: \bar{x}\right\}$ and $w_{4}=\left\{t_{3}:\right.$ $\left.\bar{x}, t_{4}: x\right\}$. Similarly, $p\left(w_{3}\right)=f_{1} \times \overline{f_{1}}$ and $p\left(w_{4}\right)=\overline{f_{1}} \times f_{1}$. We have

$$
p\left(t_{3}: x\right)=p\left(w_{4} \mid Q I_{2}\right)=f_{1} \overline{f_{1}} /\left(f_{1} \overline{f_{1}}+\overline{f_{1}} f_{1}\right)=1 / 2 .
$$

From (7), we obtain

$$
\begin{aligned}
f_{1} & =\left[f_{1} \overline{f_{2}} /\left(f_{1} \overline{f_{2}}+\overline{f_{1}} f_{2}\right)+1\right] / 3 \\
& =\left[f_{1}\left(1-f_{2}\right) /\left(f_{1}\left(1-f_{2}\right)+\left(1-f_{1}\right) f_{2}\right)+1\right] / 3 .
\end{aligned}
$$


Similarly, since $Q I_{1}$ contains one tuple $t_{2}$ matching $s_{2}$ and $Q I_{3}$ contains two tuples $t_{5}, t_{6}$ matching $s_{2}$,

$$
\begin{aligned}
f_{2} & =\left[1 \times p\left(t_{2}: x\right)+2 \times p\left(t_{5}: x\right)\right] /(1+2) \\
& =\left[\overline{f_{1}} f_{2} /\left(f_{1} \overline{f_{2}}+\overline{f_{1}} f_{2}\right)+0\right] / 3 \\
& =\left[\left(1-f_{1}\right) f_{2} /\left(f_{1}\left(1-f_{2}\right)+\left(1-f_{1}\right) f_{2}\right)\right] / 3 .
\end{aligned}
$$

With these two equations involving two variables, we adopt Newton's method to solve for these variables.

Finally, we obtain $f_{1}=0.666667$ and $f_{2}=0.000000$. Thus, we derive $\overline{f_{1}}=0.333333$ and $\overline{f_{2}}=1.000000$.

\subsection{Pruning Attribute Sets}

The adversary may choose to attack with as many attribute sets as possible. Although there are many attribute sets in the anonymized data, it is not always true that the global distribution of each attribute set is reliable because if the derived global distribution is based on a small sample or a small set of tuples matching the same signature, the distribution is not accurate. For example, consider attribute set $\mathcal{A}=$ "Nationality" and the signature \{"Malaysian"\}. Suppose there are only a few Malaysians, says 10 Malaysians, in the published table $T^{*}$. Intuitively, 10 Malaysians cannot represent a meaningful global distribution. We will make use of the sample size studied in the literature of statistics to determine whether the distribution is reliable or not. The adversary can launch an attack only based on reliable distributions.

Based on studies in statistics [Toivonen 1996], we use the following theorem to determine the acceptable sample size (the size of the set that contains the tuples matching the same signature $s$ ). Let $S$ be a random sample of tuples for a signature $s$, and $p$ be the expected fraction of tuples in $S$ with the sensitive value $x$. Let $\widetilde{p}$ be the observed fraction of tuples with the sensitive value $x$ in the sample $S$. Then the following theorem applies.

TheOREM 4.2 (SAMPle Size [TOIVONEN 1996]). Given an error parameter $\epsilon \geq 0$ and a confidence parameter $\sigma \geq 0$, if random sample $S$ has size $|S| \geq \frac{1}{2 \epsilon^{2}} \ln \frac{2}{\sigma}$, the probability that $|\widetilde{p}-p|>\epsilon$ is at most $\sigma$.

In case the sample size is not large enough to satisfy the error bound, then uniform distribution will be assumed. The sample size satisfies the monotonicity property. Formally, without loss of generality, assume that there are $u$ attributes, namely $A_{1}, \ldots, A_{u}$. Let $v_{1} \in A_{1}, \ldots, v_{u} \in A_{u}$. Let $y\left(v_{1}, \ldots, v_{i}\right)$ be the number of tuples with attributes $\left(A_{1}, \ldots, A_{i}\right)$ equal to $\left(v_{1}, \ldots, v_{i}\right)$. Given a positive integer $J$, if $y\left(v_{1}, \ldots, v_{i}\right)<J$, then $y\left(v_{1}, \ldots, v_{i}, v_{i+1}\right)<J$. With this monotonicity property, whenever we find that the sample size of $y\left(v_{1}, \ldots, v_{i}\right)$ is not large enough, we do not need to count the number of the tuples with values $v_{1}, \ldots, v_{i+1}$ because $y\left(v_{1}, \ldots, v_{i}, v_{i+1}\right)$ is also not large enough. Thus, this can help to prune the search space.

\subsection{Algorithm}

In this section, we will describe how to compute the set $\mathcal{G}$ of all global distributions of multiple attribute sets with the use of the sample size just described. The steps are shown in Algorithm 1.

In the algorithm, Step 1 is to find all signatures with sufficient sample size for each attribute set $\mathcal{A}$. Similar to frequent pattern mining, this step is typically computed within a reasonable time. Let $\alpha$ be the time for this step. After we have determined 


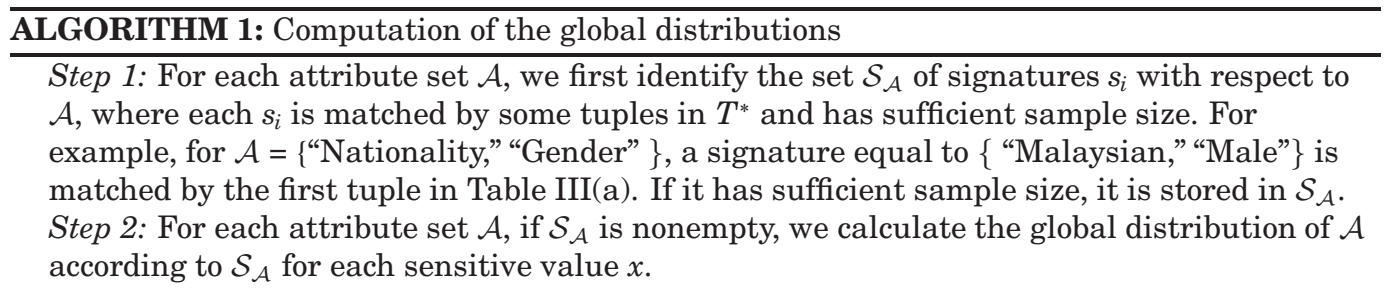

the sample sizes, $\mathcal{G}$ is used to store the global distributions of all attribute sets, each of which contains signatures with sufficient sample size.

Step 2 is to calculate the global distribution of $\mathcal{A}$ according to nonempty $\mathcal{S}_{\mathcal{A}}$ for each attribute set $\mathcal{A}$. In other words, it finds each global distribution in $\mathcal{G}$. As described in Section 4.1, for a particular global distribution, we formulate $m$ equations with $m$ variables, where $m$ is the total number of signatures for $A$. The average number of terms in each equation is $O\left(N \cdot\left|\mathcal{W}_{k}\right| \cdot\left|\mathcal{Q I}_{s_{i}}\right|\right)$, where $N$ is the average QI-group size, $\left|\mathcal{W}_{k}\right|$ is the average number of possible worlds in a QI-group $Q I_{k}$, and $\left|\mathcal{Q} \mathcal{I}_{s_{i}}\right|$ is the average number of QI-groups with tuples matching a signature $s_{i}$. If Newton's method takes $\beta$ time to find a solution, the computation for a global distribution takes $O\left(m \cdot N \cdot\left|\mathcal{W}_{k}\right| \cdot\left|\mathcal{Q I}_{s_{i}}\right|+\beta\right)$ time. Since there are $|\mathcal{G}|$ global distributions, Step 2 takes $O\left(|\mathcal{G}| \cdot\left(m \cdot N \cdot\left|\mathcal{W}_{k}\right| \cdot\left|\mathcal{Q I}_{s_{i}}\right|+\beta\right)\right)$ time.

Thus, the total running time is $O\left(\alpha+|\mathcal{G}| \cdot\left(m \cdot N \cdot\left|\mathcal{W}_{k}\right| \cdot\left|\mathcal{Q I}_{s_{i}}\right|+\beta\right)\right)$. Note that the values of $m, N,\left|\mathcal{W}_{k}\right|$ and $\left|\mathcal{Q I}_{s_{i}}\right|$ are small and the complexity is dominated by $|\mathcal{G}|$ and $\beta$. But, as the attribute set size increases, the sample size quickly becomes insufficient, and so $|\mathcal{G}|$ is typically well-behaved.

From our experiments, in all of our cases, Step 2 with the system of $m$ equations can be solved in a relatively short time. So, $\beta$ is also a reasonable value. For the benchmark dataset, adult, foreground knowledge can be mined within 12 minutes in all our experiments.

The probabilistic analysis is similar in nature to that studied for uncertain databases [Antova et al. 2007; Burdick et al. 2005, 2007]. This computational complexity is in fact much smaller than these previous works. In Antova et al. [2007], all results are returned within 3 hours. The reason is that Burdick et al. [2005, 2007] and Antova et al. [2007] analyze the possible worlds based on the entire uncertain table (which can be regarded as a single large QI-group) while we analyze the possible worlds based on a single small QI-group (which is typically smaller than the entire table).

\subsection{Discussion}

We have just discussed how to find the global distribution from the published table. One may argue that the global distribution $\widetilde{\mathcal{G}}$ found from the published table is just an approximation of the true global distribution $\mathcal{G}_{o}$ found from the original table. Thus, the privacy breaches found in Section 3 according to $\widetilde{\mathcal{G}}$ are invalid. However, we disagree with this argument for the following reasons.

First, since the adversary does not have the true global distribution $\mathcal{G}_{0}$ (because s/he has not seen the original table), the best adversary's knowledge about the global distribution is $\widetilde{\mathcal{G}}$.

Second, an adversary with $\widetilde{\mathcal{G}}$ is more powerful and more sophisticated than another adversary without any knowledge about the global distribution. The former adversary is what we are studying in this article and can breach individual privacy as discussed in Section 3, while the latter adversary is the normal adversary studied in the privacy 
literature [Machanavajjhala et al. 2006; Wong et al. 2007; Xiao and Tao 2006] and cannot breach any individual privacy found by the former adversary.

Third, previous work considers adversaries that are equipped with some external knowledge about the dataset (background knowledge). Here, we consider foreground knowledge attacks that can succeed without this external knowledge. Thus, our attacker is more sophisticated and powerful.

\section{EMPIRICAL STUDY}

A Pentium IV 2.2GHz PC with 1GB RAM was used to conduct our experiment. The algorithm was implemented in $\mathrm{C} / \mathrm{C}++$. We adopted the publicly available dataset, Adult Database, from the UCIrvine Machine Learning Repository [Blake and Merz 1998]. This dataset (5.5MB) was also adopted by LeFevre et al. [2005], Machanavajjhala et al. [2006], Wang et al. [2004], Fung et al. [2005], and Wong et al. [2007]. We used a configuration similar to LeFevre et al. [2005], Machanavajjhala et al. [2006], and Wong et al. [2007]. The records with unknown values were first eliminated resulting in a dataset with 45,222 tuples (5.4MB). Nine attributes were chosen in our experiment, namely Age, Work Class, Marital Status, Occupation, Race, Sex, Native Country, Salary Class, and Education. By default, we chose the first five attributes and the last attribute as the quasiidentifer and the sensitive attribute, respectively. Similar to Wong et al. [2007], in attribute "Education," all values representing the education levels before "secondary" (or "9th-10th") such as "1st-4th," "5th-6th," and "7th-8th" are regarded as a sensitive value set, where an adversary checks whether each individual is linked to this set more than $1 / r$, where $r$ is a parameter.

There are $3.46 \%$ tuples with education levels before "secondary." We set $\epsilon=0.01$ and $\sigma=0.9$ for sampling. That is, the allowed relative error of sampling is $1 / 3.46=$ $28.90 \%$, which is considered large. A larger allowed error means less attribute sets can be pruned. Since there is a set $\mathcal{G}$ of multiple global distributions $G$, we can calculate $p(t: x)$ for different $G$ 's and different $x$ 's. We take the greatest such value to report as the probability that individual $t$ is linked to some sensitive value since this corresponds to the worst case privacy breach.

\subsection{Privacy Breach in $l$-diverse Tables}

In this section, we will show that foreground knowledge attack is successful in the published data generated from the benchmark dataset, adult, by a well-known privacy algorithm, Anatomy [Xiao and Tao 2006]. We set $l=r$, where $l$ is the parameter of $l$-diversity used in Anatomy. We implemented the formula in Section 3 to calculate the probability of a privacy breach and the formula in Section 4 to find the global distribution from the published data. If a tuple that appears in the published data is identified as a privacy breach by our algorithm, it is said to be a problematic tuple. The tuples linking to sensitive values in the original table are called sensitive tuples. In this case study, we evaluate privacy breaches with the following five measurements.

(1) The proportion of problematic tuples among sensitive tuples, (this is the recall in IR research);

(2) the proportion of nonsensitive tuples that are wrongly identified as problematic tuples by our algorithm;

(3) the average probability by which individual privacy is breached among all sensitive tuples;

(4) the average absolute difference between $1 / r$ and the probability by which individual privacy is breached among all sensitive tuples;

(5) the average square difference between $1 / r$ and the probability by which individual privacy is breached among all sensitive tuples. 
We have conducted experiments with variation of $r$ and variation of the QI size. (1) Variation of $r$. When $r=2$ with default settings, the average probability that individual privacy breaches among all sensitive tuples is $0.8917(>1 / 2)$. When $r$ is increased to 4 , it becomes $0.4640(>1 / 4)$. When $r$ increases, there is a higher chance that a tuple forms a QI-group with other tuples. Thus, the average size of QI-groups is larger. Thus, the average probability of privacy breaches decreases.

We also studied the proportion of problematic tuples among all sensitive tuples and the proportion of nonsensitive tuples wrongly identified as privacy breaches. We found that, in most cases, more than $99 \%$ of sensitive tuples have privacy breaches and less than $6 \%$ of nonsensitive tuples are wrongly identified. This shows that the problem caused by the foreground knowledge is quite serious. The small percentage of false alarms confirms our concern that foreground knowledge can be used as a reliable information source for the adversary.

We also measured the average absolute difference between $1 / r$ and the probability by which individual privacy is breached among all sensitive tuples. When $r=2$, it is equal to 0.3917 . When $r$ is increased to 4 , it becomes 0.2140 . This is because, when $r$ is larger, the size of a QI-group is larger and thus it is more difficult to have privacy breaches. Thus, the average absolute difference is smaller. Similarly, we measured the average square difference between $1 / r$ and the probability by which individual privacy is breached among all sensitive tuples. The trend is similar. When $r$ is 2 , it is equal to 0.1810 . When $r$ becomes 4 , it is equal to 0.0675 .

(2) Variation of the QI size: When the QI size is equal to 3 with default settings, where $r=2$, the average probability causing privacy breaches is 0.80307 . When the size is increased to 8 , it becomes 0.943526 . This is because when there are more QI attributes, it is more likely that a QI attribute (or attribute set) gives a global distribution that can lead to privacy breaches.

We also have a case study on the published data generated by Anatomy. Suppose the QI attributes chosen are Age, Marital Status, and Occupation and the sensitive attribute is Education. In the original data, there are the following 2 tuples.

\begin{tabular}{|c|c|c|c|}
\hline Age & Marital Status & Occupation & Education \\
\hline 39 & Never-married & Adm-clerical & Bachelors \\
\hline 20 & Married-civ-spouse & Craft-repair & 5th-6th \\
\hline
\end{tabular}

Suppose the objective of Anatomy is 2-diversity. Since "5th-6th" is a sensitive value, Anatomy forms a QI-group containing these two tuples. However, from the global distribution derived from the published data with respect to attribute Occupation, the probability that an individual with Occupation="Adm-clerical" is linked to a low education is only 0.02 but the probability that an individual with Occupation="Craft-repair" is linked to a low education is 0.04 . Since there is a significant difference in global distribution of attribute Occupation, the probability that the second tuple is linked to a low education is 0.67 (which is greater than 0.5).

It is noted that the global distribution derived from the published data matches the real situation that "Adm-clerical" jobs require higher educations than "Craft-repair." In other words, the foreground knowledge can help the adversary to breach individual privacy. More specifically, let us check whether the real global distribution derived from the original table is similar to the global distribution derived from the published data. From the original table, the probability that an individual with Occupation="Admclerical" is linked to a low education is only 0.01 but the probability that an individual with Occupation="Craft-repair" is linked to a low education is 0.04 . We observe that this global distribution is similar to that derived from the published data. 
With our default experimental setting using sufficient sample size, for 2-diversity, the average relative error of the global probabilities derived from the published data $=0.7 \%$, which achieves $99.3 \%$ accuracy. For 10-diversity, the error increases to $5.26 \%$ where the accuracy is $94.74 \%$. It shows that statistically the accuracy is very high. In other words, the foreground knowledge derived from the published data is quite accurate compared with the knowledge derived from the original table.

In all our experiments, privacy breaches can be found within 12 minutes, which shows that foreground knowledge attack can easily be realized.

\subsection{Privacy Breach in Other Privacy Models}

We studied privacy breaches with four algorithms, Anatomy [Xiao and Tao 2006], MASK [Wong et al. 2007], Injector [Li and $\mathrm{Li} 2008$ ] and $t$-closeness [ $\mathrm{Li}$ and $\mathrm{Li}$ 2007]. They are selected because they consider $l$-diversity or similar privacy requirements, so we need only set $l=r$. For Anatomy, we set $l=r$. For MASK, the parameters $k$ and $m$ used in MASK are set to $r$. For Injector, the parameters minConf, minExp, and $l$ are set to 1, 0.9, and $r$, respectively, which are the default settings in Li and Li [2008]. For $t$-closeness, similar to $\mathrm{Li}$ and $\mathrm{Li}$ [2007], we set $t=0.2$, and as in $\mathrm{Li}$ and $\mathrm{Li}$ [2007], algorithm Incognito [LeFevre et al. 2005] is adopted in the computation. We evaluate the algorithms in terms of four measurements: (1) time for mining foreground knowledge, (2) execution time, (3) the proportion of problematic tuples among all sensitive tuples, (4) the average of the greatest difference in the global probabilities in each QI-group (In our figures, we label this as average value of $\triangle$ ), and (5) the relative error ratio in answering an aggregate query as in Xiao and Tao [2006], Wong et al. [2007], and $\mathrm{Li}$ and $\mathrm{Li}$ [2008], by the published data. For each measurement, we conducted the experiments 100 times and took the average.

We do not report the time for finding privacy breaches because the time is very short (within a few minutes). For the sake of space, since the proportion of nonsensitive tuples wrongly identified for privacy breaches is small (less than 10\%), we do not report them here.

Let us explain measurements (4) and (5). (4) Consider that a QI-group $Q I_{k}$ contains two tuples matching signatures $s_{i}$ and $s_{j}$, respectively. Suppose $p\left(s_{i}: x\right)$ is the greatest global probability and $p\left(s_{j}: x\right)$ is the smallest in the QI-group. The value of $\triangle$ in $Q I_{k}$ is equal to $p\left(s_{i}: x\right)-p\left(s_{j}: x\right)$. The average value of $\triangle$ is taken among all QI-groups and all attribute sets $\mathcal{A}$ with sufficient samples. (5) The relative error ratio measures the utility of the published data. We adopt all query parameters in Xiao and Tao [2006], Wong et al. [2007], and $\mathrm{Li}$ and $\mathrm{Li}$ [2008]. For each evaluation, we performed 10,000 queries and reported the average relative error ratio.

We conducted the experiments by varying two factors: (1) the QI size, and (2) $r$.

Figure 1 and Figure 2 show the results when $r$ is set to 2 and 10, respectively. Figure 1(a) shows that the time for mining foreground knowledge increases with the QI size because the algorithm needs to process more attribute sets. Figure 1(b) shows that the execution time increases with the size of QI because the algorithms have to process more QI attributes.

Figure 1(c) shows that the proportion of problematic tuples among sensitive tuples increases with QI size. With a larger QI size, there is a higher chance that individual privacy breaches due to more attributes that can be used to construct the global distributions. MASK has fewer privacy breaches compared with Anatomy and Injector because the side-effects of the minimization of QI values in each QI-group adopted in MASK makes the difference in the global distribution among all tuples in each QIgroup smaller. Thus, the number of individuals with privacy breaches is smaller. It is noted that there is no violation in $t$-closeness. The reason why $t$-closeness has no 


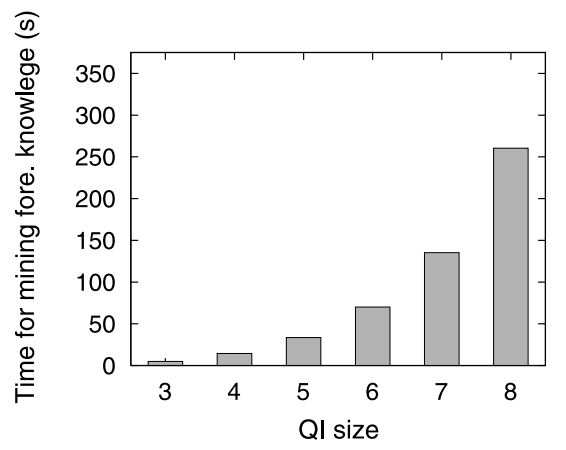

(a)

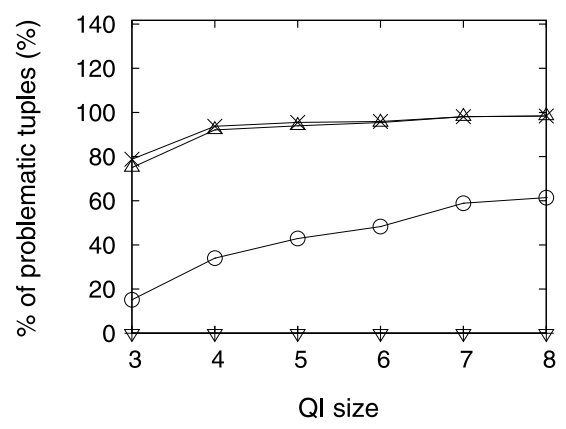

(c)

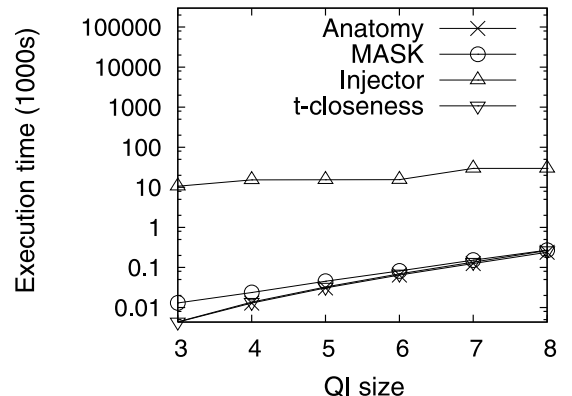

(b)

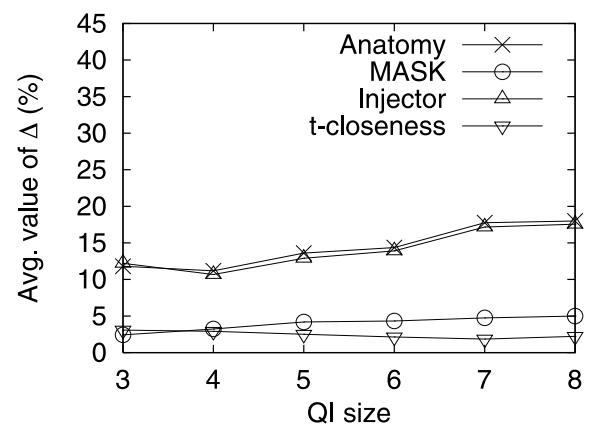

(d)

Fig. 1. Effect of QI size $(r=2)$.

privacy breaches is due to the large QI-groups formed by global recoding with respect to value $r(=2)$. The average size of the QI-group in the table satisfying $t$-closeness is at least 4000 and the utility of the table is low. It is noted that parameter $t$ is independent of parameter $r$. We will show that $t$-closeness has privacy breaches when $r=10$.

In Figure 1(d), when the QI size increases, the average value of $\Delta$ with respect to every attribute set increases, as shown in Figure 1(d). The average value of $\triangle$ is largest in Anatomy and Injector, and third largest in MASK. This is because Anatomy and Injector do not take the global distribution directly into consideration for merging, but $M A S K$ does indirectly during the minimization of QI values.

Figure 3(a) shows that the average relative error of $t$-closeness is the largest since it forms large QI-groups by global recoding, which introduces many errors and thus reduces the utility of the published data.

We have also conducted experiments when $r=10$, as shown in Figure 2. The results are also similar. But, the time for mining foreground knowledge is larger. Since $r$ is larger and thus $1 / r$ is smaller, the average value of $\Delta$ is smaller when $r=10$. Also, when $r=10$, there are privacy breaches for $t$-closeness in Figure 2(c) because there is a higher privacy requirement when $r=10$ and thus the size of the QI-group is not large enough for protection.

\section{RELATED WORK}

With respect to attribute types considered for data anonymization, there are two branches of studying. The first branch is anonymization according to the QI attributes. A typical model is $k$-anonymity [Aggarwal et al. 2005; LeFevre et al. 2005]. The other 


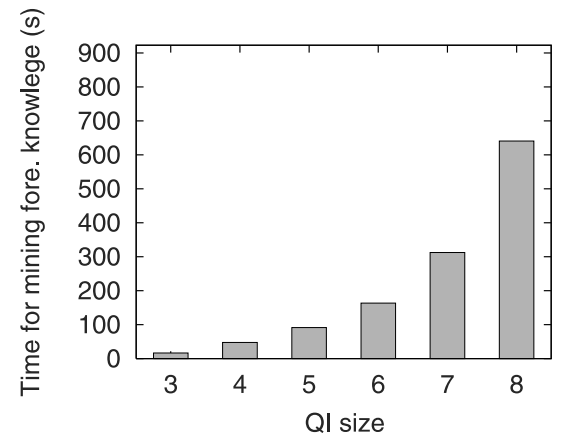

(a)

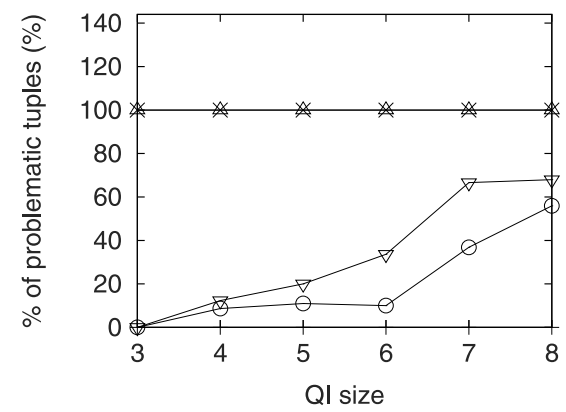

(c)

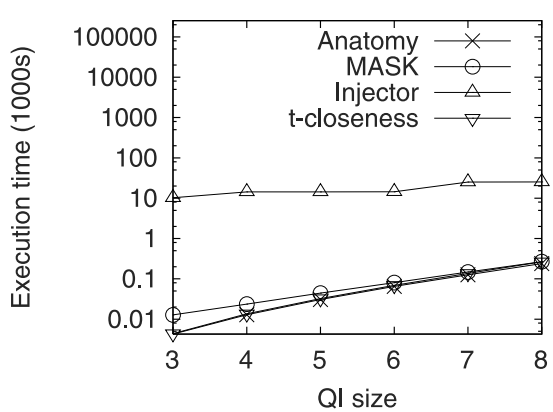

(b)

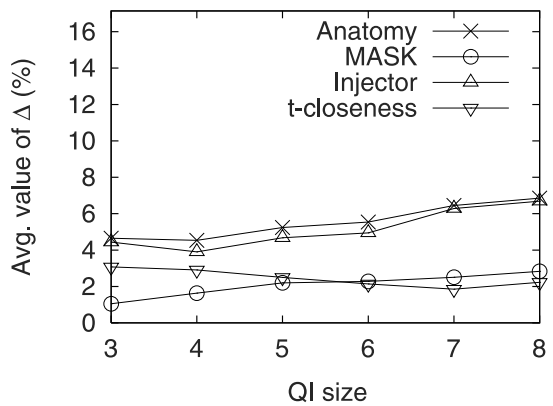

(d)

Fig. 2. Effect of QI size $(r=10)$.

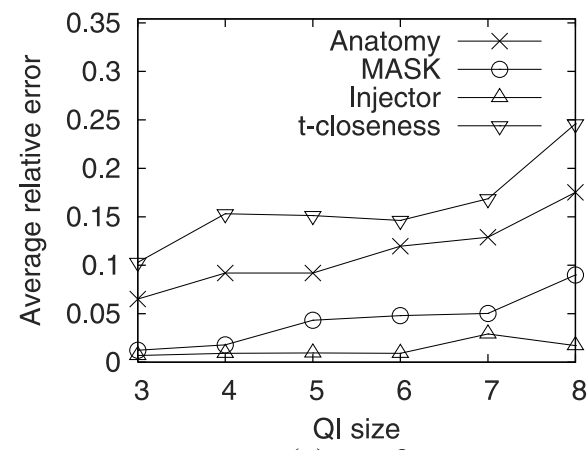

(a) $r=2$

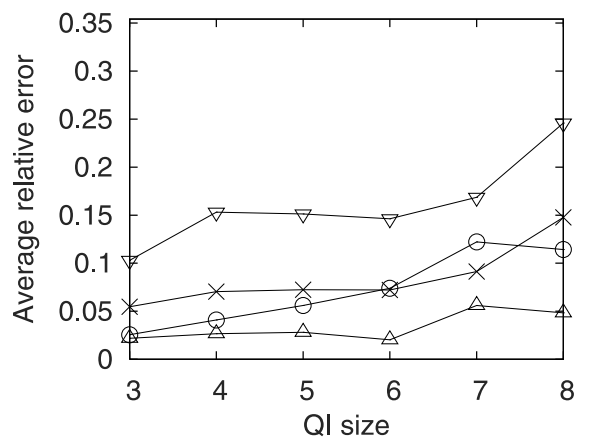

(b) $r=10$

Fig. 3. Effect of QI size on average relative error.

branch is the consideration of both quasiidentifier attributes and sensitive attributes. Some examples are Machanavajjhala et al. [2006], Wong et al. [2006], Li and Li [2007, 2008], and Brickell and Shmatikov [2008]. In this article we focus on that branch. We want to check whether the probability that each individual is linked to any sensitive value is at most a given threshold.

$l$-diversity [Machanavajjhala et al. 2006] proposes a model where $l$ is a positive integer and each QI-group contains $l$ well-represented sensitive values. For $t$-closeness [Li and $\mathrm{Li}$ 2007], the distribution in each QI-group in $T^{*}$ with respect to the sensitive attribute is roughly equal to the distribution of the entire table $T^{*}$. Given a real 
number $\alpha \in[0,1]$ and a positive integer $k,(\alpha, k)$-anonymity [Wong et al. 2006] maintains that for each QI-group $Q I$, the number of tuples in $Q I$ is at least $k$ and the frequency (in fraction) of each sensitive value in $Q I$ is at most $\alpha$.

In the literature, different kinds of background knowledge are considered [Aggarwal et al. 2006; Ganta et al. 2008; Kifer and Gehrke 2006; Li and Li 2008; Li et al. 2009; Machanavajjhala et al. 2006; Martin et al. 2007; Wong et al. 2007, 2010]. Kifer and Gehrke [2006] propose that the statistics of some attributes such as age and zipcode can also be available to the public. Martin et al. [2007] consider other background knowledge in the form of implications. Wong et al. [2007] discover that the minimality principle of the anonymization algorithm can also be used as background knowledge. Li et al. [2009] propose using the kernel estimation method to mine the background knowledge from the original table. Wong et al. [2010] study how to use probabilistic distribution-based background knowledge for anonymization. Ganta et al. [2008] describe that there are many tables published from different sources containing overlapping individuals.

$\mathrm{Li}$ and $\mathrm{Li}$ [2008] find that association rules can be mined from the original table and thus can be used for privacy protection during anonymization. In Aggarwal et al. [2006], the problem of privacy attack by adversarial association rule mining is investigated. Hence, the association rules are the foreground knowledge. However, as pointed out in Silverstein et al. [1997], association rules used in Li and Li [2008] and Aggarwal et al. [2006] can contradict the true statistical properties. Also the solution in Aggarwal et al. [2006] is to invalidate the rules, but this will violate the data mining objectives of data publication.

A recent work [Aggarwal 2008] proposes generating a table in the form of an uncertain data model. However, this work considers only $k$-anonymity, which ignores any sensitive attribute.

Recently, Kifer [2009] proposed using the concepts of exchangeability and deFinetti's theorem to reason about privacy attacks. Kifer [2009] proposes using a machine learning model, Naive Bayes, to model the foreground knowledge derived from the published table. The work in Kifer [2009] is different from ours in the following ways. First, Kifer [2009] makes an independence assumption among QI attributes when the foreground knowledge is considered. We do not have such an assumption because the foreground knowledge is expressed in the form of global distributions with respect to different attribute sets $\mathcal{A}$, where $\mathcal{A}$ is of any size and can express the correlations among attributes in $\mathcal{A}$. Second, the modeling of foreground knowledge in Kifer [2009] is different from ours. Kifer [2009] adopts the Naive Bayes model to express the foreground knowledge, where the probability of a certain assignment of an individual to a sensitive value is based on the assumption that all distributions of the linkage probabilities are the same. However, this assumption has no basis. We also adopt Bayesian probability, but our prior knowledge in the Bayesian probability is the linkage probability based on the given table, and the posterior probability is the linkage probability on the condition that the given tuple is within a certain anonymized group. Our prior knowledge is hence not based on an unrealistic assumption. Third, Kifer [2009] shows the attack in the published table generated by a particular algorithm, Anatomy, for a particular privacy requirement, $l$-diversity. But, we show empirically the attacks in published tables generated by not only Anatomy [Xiao and Tao 2006] but also other existing algorithms (e.g., MASK [Wong et al. 2007] and Injector [Li and Li 2008]) for many privacy requirements like $l$-diversity, $m$-confidentiality and $t$-closeness. It is important to show how privacy breaches occur in other algorithms in the literature.

Instead of publishing an anonymized table, Zhu et al. [2009] consider publishing the data mining results in the form of association rules that can be obtained from the original table. In Zhu et al. [2009], the main focus is to derive sensitive information 
from a set of association rules. Zhu et al. [2009] differs from our approach since it does not study how to derive sensitive information from the anonymized table.

\section{CONCLUSION}

In this article, we point out a fundamental privacy breach problem that has been overlooked in the past. With consideration of the utility of the anonymized table, group based anonymization suffers from privacy breaches. Our experiments show that existing well-known privacy models Anatomy, MASK, Injector, and $t$-closeness suffer from serious privacy breaches in a benchmark dataset. For future work, we plan to study how to anonymize the data to defend against foreground knowledge attack. In our experiment, we observe that the chance of privacy breaches is lower if each group contains tuples with similar global probabilities. Thus, forming QI-groups with similar tuples is one possible strategy. Another future project is to study the effect of background knowledge that may be possessed by the adversary.

\section{ACKNOWLEDGMENTS}

We are grateful to the anonymous reviewers for their constructive comments on this article.

\section{REFERENCES}

AGGARWAL, C. C. 2008. On unifying privacy and uncertain data models. In Proceedings of the International Conference on Data Engineering (ICDE). 386-395.

AgGarwal, C. C., Pei, J., AND Zhang, B. 2006. On privacy preservation against adversarial data mining. In Proceedings of the ACM SIGKDD Conference on Knowledge Discovery and Data Mining (KDD). 510-516.

Aggarwal, G., Feder, T., Kenthapadi, K., Motwani, R., Panigrahy, R., Thomas, D., And Zhu, A. 2005. Anonymizing tables. In Proceedings of the International Conference on Database Theory (ICDT). $246-258$.

Antova, L., Koch, C., ANd Olteanu, D. 2007. $10^{10^{6}}$ worlds and beyond: Efficient representation and processing of incomplete information. In Proceedings of the International Conference on Data Engineering (ICDE). 606-615.

BLAKE, E. K. C. AND MERZ, C. J. 1998. UCI repository of machine learning databases. http://www.ics.uci.edu/ mlearn/MLRepository.html.

BRickell, J. AND Shmatikov, V. 2008. The cost of privacy: Destruction of data-mining utility in anonymized data publishing. In Proceedings of the ACM SIGKDD Conference on Knowledge Discovery and Data Mining (KDD). 70-78.

Burdick, D., DeshPande, P., JAYRAm, T., RAmakrishnan, R., AND VAithyANATHAN, S. 2005. Olap over uncertain and imprecise data. In Proceedings of the International Conference on Very Large Databases (VLDB). 123-144.

Burdick, D., Doan, A., Ramakrishnan, R., and Vaithyanathan, S. 2007. Olap over imprecise data with domain constraints. In Proceedings of the International Conference on Very Large Databases (VLDB). 39-50.

Chapra, S. C. AND CANAle, R. P. 2002. Numerical Methods for Engineers. McGraw-Hill, 4th ed.

Cheng, R., Chen, J., Mokbel, M., And Chow, C. 2008. Probabilistic verifiers: Evaluating constrained nearest-neighbor queries over uncertain data. In Proceedings of the International Conference on Data Engineering (ICDE). 973-982.

FUnG, B. C. M., WANG, K., AND YU, P. S. 2005. Top-down specialization for information and privacy preservation. In Proceedings of the International Conference on Data Engineering (ICDE). 205-216.

Ganta, S. R., KASIVISWANATHAN, S. P., AND SMith, A. 2008. Composition attacks and auxiliary information in data privacy. In Proceedings of the ACM SIGKDD Conference on Knowledge Discovery and Data Mining (KDD). 265-273.

IMIELINSKI, T. AND LIPSKI, JR., W. 1984. Incomplete information in relational databases. In J. ACM. $761-791$.

KIFER, D. 2009. Attacks on privacy and Definetti's theorem. In Proceedings of the ACM SIGMOD International Conference on Management of Data. 127-138. 
KIfER, D. AND GEHRKe, J. 2006. Injecting utility into anonymized datasets. In Proceedings of the ACM SIGMOD International Conference on Management of Data. 217-228.

LeFevre, K., DeWitt, D. J., AND RAmakrishnan, R. 2005. Incognito: Efficient full-domain kanonymity. In Proceedings of the ACM SIGMOD International Conference on Management of Data. 49-60.

LI, N. AND LI, T. 2007. $t$-closeness: Privacy beyond $k$-anonymity and $l$-diversity. In Proceedings of the International Conference on Data Engineering (ICDE). 106-115.

LI, T. AND LI, N. 2008. Injector: Mining background knowledge for data anonymization. In Proceedings of the International Conference on Data Engineering (ICDE). 446-455.

LI, T., LI, N., AND ZHANG, J. 2009. Modeling and integrating background knowledge in data anonymization. In Proceedings of the International Conference on Data Engineering (ICDE). 6-17.

MachanavajJhala, A., Gehrke, J., AND Kifer, D. 2006. $l$-diversity: Privacy beyond $k$-anonymity. In Proceedings of the International Conference on Data Engineering (ICDE). 24.

Martin, D. J., Kifer, D., MachanavajJhala, A., And Gehrke, J. 2007. Worst-case background knowledge for privacy-preserving data publishing. In Proceedings of the International Conference on Data Engineering (ICDE). 126-135.

Nergiz, M. E. AND Clifton, C. 2007. Thoughts on k-anonymization. In Proceedings of International Conference on Data Engineering Workshops. 622-645.

Silverstein, C., Motwani, R., AND Brin, S. 1997. Beyond market baskets: Generalizing association rules to correlations. In Proceedings of the ACM SIGMOD International Conference on Management of Data. 265-276.

SweEney, L. 2002. k-anonymity: A model for protecting privacy. Inter. J. Uncertainty, Fuzziness Knowl. Based Syst. 10, 5, 557-570.

Toivonen, H. 1996. Sampling large databases for association rules. In Proceedings of the International Conference on Very Large Databases (VLDB). 134-145.

WANG, K., YU, P. S., AND ChaKRABorTY, S. 2004. Bottom-up generalization: A data mining solution to privacy protection. In Proceedings of the IEEE International Conference on Data Mining (ICDM). 249-256.

Wong, R., FU, A., WANG, K., AND PEI, J. 2007. Minimality attack in privacy preserving data publishing. In Proceedings of the International Conference on Very Large Databases (VLDB). 543-554.

WonG, R., LI, J., FU, A., AND WANG, K. 2006. (alpha, k)-anonymity: An enhanced k-anonymity model for privacy-preserving data publishing. In Proceedings of the ACM SIGKDD Conference on Knowledge Discovery and Data Mining (KDD). 754-759.

Wong, R. C.-W., Fu, A. W.-C., WAng, K., XU, Y., Pei, J., AND Yu, P. 2010. Probabilistic inference protection on anonymized data. In Proceedings of the IEEE International Conference Data Mining (ICDM). 1127-1132.

XIAo, X. And TaO, Y. 2006. Anatomy: Simple and effective privacy preservation. In Proceedings of the International Conference on Very Large Databases (VLDB). 139-150.

XIAO, X. AND TAO, Y. 2007. $m$-invariance: Towards privacy preserving re-publication of dynamic datasets. In Proceedings of the ACM SIGMOD International Conference on Management of Data. 689-700.

Zhang, Q., Koudas, N., SRIVAstava, D., AND Yu, T. 2007. Aggregate query answering on anonymized tables. In Proceedings of the International Conference on Data Engineering (ICDE). 116-125.

ZHU, Z., WANG, G., AND DU, W. 2009. Deriving private information from association rule mining results. In Proceedings of the International Conference on Data Engineering (ICDE). 18-29.

Received May 2009; revised October 2009, April 2010, September 2010; accepted December 2010 